\title{
The Cyclotomic Numbers of Order Fifteen
}

\author{
By Nicholas Buck, Lones Smith, Blair K. Spearman, \\ and Kenneth S. Williams*
}

Dedicated to Daniel Shanks on the occasion of his 70 th birthday

\begin{abstract}
Explicit formulae are obtained for the cyclotomic numbers of order 15.
\end{abstract}
1. Introduction and Notation. Let $p=15 f+1$ be a prime, so that $f$ is even. Let $g$ be a fixed primitive root of $p$. The index of $k \not \equiv 0(\bmod p)$, written ind $k$, is the unique integer $m$ such that $k \equiv g^{m}(\bmod p), 0 \leqslant m \leqslant p-2$. For integers $i$ and $j$ $(0 \leqslant i, j \leqslant 14)$ the cyclotomic number $(i, j)_{15}$ of order 15 is defined to be the number of integers $k(2 \leqslant k \leqslant p-1)$ which satisfy

$$
\operatorname{ind}(k-1) \equiv i(\bmod 15), \quad \text { ind } k \equiv j(\bmod 15) .
$$

The purpose of this paper is to give explicit formulae for the cyclotomic numbers of order 15. Formulae for the cyclotomic numbers of orders 2, 3, 4, 5, 6 [4]; 7 [11]; 8 [10]; 9 [2]; 10 [18]; 11 [12]; 12 [19]; 14 [13]; 16 [17], [6]; 18 [2]; 20 [15]; 24 [7], are already known.

Closely related to the cyclotomic numbers $(h, k)_{15}$ are the Jacobi sums $J_{15}\left(\beta^{m}, \beta^{n}\right)$ of order 15, defined for integers $m$ and $n$ by

$$
J_{15}\left(\beta^{m}, \beta^{n}\right)=\sum_{k=2}^{p-1} \beta^{m \text { ind } k+n \text { ind }(1-k)},
$$

where $\beta=\exp (2 \pi i / 15)$. The basic properties of these sums are given in Section 2 . The study of the Jacobi sums of order 15 was begun by L. E. Dickson [5] in 1935 and completed by J. B. Muskat [14] in 1968.

In this paper we use Dickson's and Muskat's evaluations of the Jacobi sums of order 15 (see Sections 3 and 4) to obtain the values of the Dickson-Hurwitz $B_{15}(i, v)$ of order 15 defined by

$$
B_{15}(i, v)=\sum_{h=0}^{14}(h, i-v h)_{15}
$$

see Section 5. In Section 6, a special case of a theorem of Friesen, Muskat, Spearman, and Williams [8, Theorem 7] is used to express each cyclotomic number in terms of the Dickson-Hurwitz sums, and then, using the values for the DicksonHurwitz sum obtained in Section 5, explicit formulae for the cyclotomic numbers

Received January 7, 1986; revised May 5, 1986.

1980 Mathematics Subject Classification. Primary 12C20.

* Research supported by Natural Sciences and Engineering Research Council Canada Grant A-7233. 
$(i, j)_{15}$ are derived (see Tables $\left.1-70\right)$. It turns out that each number $225(i, j)_{15}$ can be expressed as an integral linear combination of the integers $p, 1, a, b, c, d, x, u$, $v, w, b_{0}, b_{1}, b_{2}, b_{3}, b_{4}, b_{5}, b_{6}, b_{7}$, where the quantities $a, b, \ldots, b_{7}$ are given by

$$
\begin{aligned}
& \beta^{10 \text { ind } 2} J_{15}\left(\beta^{5}, \beta^{5}\right)=a+b \sqrt{-3} \\
& \beta^{10 \text { ind } 5} J_{15}\left(\beta, \beta^{4}\right)=c+d \sqrt{-15} \\
& \beta^{6 \text { ind } J_{15}}\left(\beta^{3}, \beta^{3}\right)=x+u \sqrt{-5-2 \sqrt{5}}+v \sqrt{-5+2 \sqrt{5}}+w \sqrt{5} \\
& J_{15}(\beta, \beta)=\sum_{j=0}^{7} b_{j} \beta^{j} .
\end{aligned}
$$

There are 450 sets of tables for the $(i, j)_{15}$, depending upon the values of ind 2 $(\bmod 15)$, ind $3(\bmod 5)$, ind $5(\bmod 3), c(\equiv \pm 1)(\bmod 5)$. However, as explained in Section 6 , it is only necessary to list 70 of these tables, as the remaining 380 tables can be deduced from them.

The integers $a, b, c, d, x, u, v, w$ have the following properties:

$$
\begin{gathered}
p=a^{2}+3 b^{2}, \quad a \equiv-1(\bmod 3) \\
p=c^{2}+15 d^{2}, \quad c \equiv-1(\bmod 3) \\
\left\{\begin{array}{l}
p=x^{2}+5 u^{2}+5 v^{2}+5 w^{2} \\
x w=v^{2}-u v-u^{2}, \quad x \equiv-1(\bmod 5)
\end{array}\right.
\end{gathered}
$$

We remark that $a$ is determined uniquely by $(1.8) ; c$ is determined uniquely by (1.9); and $x$ is determined uniquely by (1.10) (see [16, p. 17]).

For example, when $p=61, g=2$, we have

$$
\begin{gathered}
a=-7, \quad b=2, \quad c=-1, \quad d=-2, \\
x=4, \quad u=2, \quad v=2, \quad w=-1, \\
b_{0}=-3, \quad b_{1}=1, \quad b_{2}=4, \quad b_{3}=-6, \quad b_{4}=5, \quad b_{5}=-3, \quad b_{6}=-4, \quad b_{7}=11 .
\end{gathered}
$$

We conclude this section by remarking that all the computations for this paper were carried out on a Digital Professional 350 microcomputer. The authors would like to thank David Conibear for his help in running the programs.

2. Basic Properties of Jacobi and Gauss Sums. The Jacobi sums $J_{15}\left(\beta^{m}, \beta^{n}\right)$ have the following well-known properties (see, for example, [14, pp. 483-484]):

$$
\begin{aligned}
& J_{15}\left(\beta^{m}, \beta^{n}\right)=J_{15}\left(\beta^{n}, \beta^{m}\right)=J_{15}\left(\beta^{-m-n}, \beta^{n}\right) ; \\
& J_{15}\left(\beta^{m}, \beta^{n}\right) J_{15}\left(\beta^{-m}, \beta^{-n}\right)=p \quad \text { if } 15+m, 15+n, 15+m+n ; \\
& J_{15}\left(\beta^{m}, \beta^{n}\right)=-1 \quad \text { if } 15+m, 15 \mid n ; \text { or } 15 \mid m, 15+n ; \\
& \quad \text { or } 15+m, 15+n, 15 \mid m+n ; \\
& J_{15}\left(\beta^{m}, \beta^{n}\right)=p-2 \text { if } 15|m, 15| n .
\end{aligned}
$$

Closely related to the Jacobi sum $J_{15}\left(\beta^{m}, \beta^{n}\right)$ is the Gauss sum $G_{15}\left(\beta^{m}\right)$ defined for any integer $m$ by

$$
G_{15}\left(\beta^{m}\right)=\sum_{r=1}^{p-1} \beta^{m \text { ind } r} \zeta^{r}
$$


where $\zeta=\exp (2 \pi i / p)$. The Gauss sums $G_{15}\left(\beta^{m}\right)$ have the following properties (see, for example, $[14$, p. 484]):

$$
\begin{array}{ll}
G_{15}\left(\beta^{m}\right)=-1 & \text { if } 15 \mid m, \\
G_{15}\left(\beta^{m}\right) G_{15}\left(\beta^{-m}\right)=p & \text { if } 15+m .
\end{array}
$$

The basic relationship between these Gauss and Jacobi sums is (see, for example, [14, Eq. (3)])

$$
J_{15}\left(\beta^{m}, \beta^{n}\right)=\frac{G_{15}\left(\beta^{m}\right) G_{15}\left(\beta^{n}\right)}{G_{15}\left(\beta^{m+n}\right)} \text { if } 15+m, 15+n, 15+m+n .
$$

3. Consideration of Gauss Sums $G_{15}\left(\beta^{j}\right)$. In this section we use the DavenportHasse identity [3, Eq. $(0.9)_{1}$ ] to determine relationships among the Gauss sums $G_{15}\left(\beta^{j}\right)(j=1,2, \ldots, 14)$.

THEOREM 1. Let $p \equiv 1(\bmod 15)$ be a prime. Let $g$ be a primitive root $(\bmod p)$ and let $\beta=\exp (2 \pi i / 15)$. Then the Gauss sums $G_{15}\left(\beta^{j}\right)(j=1,2, \ldots, 14)$ are related as follows:

$$
\begin{aligned}
& G_{15}(\beta)=A, \\
& G_{15}\left(\beta^{2}\right)=B, \\
& G_{15}\left(\beta^{3}\right)=C, \\
& G_{15}\left(\beta^{4}\right)=\theta \beta^{-3 \text { ind } 3+5 \text { ind } 5} B D / C, \\
& G_{15}\left(\beta^{5}\right)=D, \\
& G_{15}\left(\beta^{6}\right)=\theta \beta^{-6 \text { ind } 3+5 \text { ind } 5} B D / A, \\
& G_{15}\left(\beta^{7}\right)=\theta \beta^{3 \text { ind } 3+5 \text { ind } 5} C D / A, \\
& G_{15}\left(\beta^{8}\right)=\theta \beta^{-3 \text { ind } 3-5 \text { ind } 5} p A / C D, \\
& G_{15}\left(\beta^{9}\right)=\theta \beta^{6 \text { ind } 3-5 \text { ind } 5} p A / B D, \\
& G_{15}\left(\beta^{10}\right)=p / D, \\
& G_{15}\left(\beta^{11}\right)=\theta \beta^{3 \text { ind } 3-5 \text { ind } 5} p C / B D, \\
& G_{15}\left(\beta^{12}\right)=p / C, \\
& G_{15}\left(\beta^{13}\right)=p / B, \\
& G_{15}\left(\beta^{14}\right)=p / A,
\end{aligned}
$$

where $\theta= \pm 1$. [The determination of $\theta$ is given in Theorem 3.]

Proof. Clearly (3.10), (3.12), (3.13), (3.14) follow from (2.7) and (3.5), (3.3), (3.2), (3.1), respectively.

Next, from the Davenport-Hasse identity, we have

$$
\begin{aligned}
& p G_{15}\left(\beta^{3}\right)=\beta^{3 \text { ind } 3} G_{15}(\beta) G_{15}\left(\beta^{6}\right) G_{15}\left(\beta^{11}\right), \\
& p G_{15}\left(\beta^{6}\right)=\beta^{6 \text { ind } 3} G_{15}\left(\beta^{2}\right) G_{15}\left(\beta^{7}\right) G_{15}\left(\beta^{12}\right),
\end{aligned}
$$

and

$$
p^{2} G_{15}\left(\beta^{5}\right)=\beta^{5 \text { ind } 5} G_{15}(\beta) G_{15}\left(\beta^{4}\right) G_{15}\left(\beta^{7}\right) G_{15}\left(\beta^{10}\right) G_{15}\left(\beta^{13}\right) .
$$

Using (3.15), (3.1), (3.3) we get

$$
G_{15}\left(\beta^{6}\right) G_{15}\left(\beta^{11}\right)=\beta^{-3 \text { ind } 3} p C / A ;
$$


using (3.16), (3.2), (3.12) we get

$$
G_{15}\left(\beta^{6}\right) / G_{15}\left(\beta^{7}\right)=\beta^{6 \text { ind } 3} B / C ;
$$

and using (3.17), (3.1), (3.5), (3.10), (3.13) we get

$$
G_{15}\left(\beta^{4}\right) G_{15}\left(\beta^{7}\right)=\beta^{-5 \text { ind } 5} B D^{2} / A \text {. }
$$

Multiplying (3.18) and (3.20) together, and using $G_{15}\left(\beta^{4}\right) G_{15}\left(\beta^{11}\right)=p$, we obtain

$$
G_{15}\left(\beta^{6}\right) G_{15}\left(\beta^{7}\right)=\beta^{-3 \text { ind } 3-5 \text { ind } 5} B C D^{2} / A^{2} \text {. }
$$

Then, multiplying (3.19) and (3.21) together, we have

$$
\left\{G_{15}\left(\beta^{6}\right)\right\}^{2}=\beta^{3 \text { ind } 3-5 \text { ind } 5} B^{2} D^{2} / A^{2},
$$

so that (3.6) follows.

From (3.6) and (3.18), (3.6) and (3.19), (3.7) and (3.20), we obtain (3.11), (3.7), (3.4), respectively. Then, from (3.6) and (2.7), we obtain (3.9) and, from (3.7) and (2.7), we obtain (3.8).

This completes the proof of Theorem 1.

4. Evaluation of Jacobi Sums $J_{15}\left(\beta^{m}, \beta^{n}\right)$. For $(k, 15)=1$ the automorphism $\sigma_{k}$ of $Q(\beta)$ is defined by $\sigma_{k}: \beta \rightarrow \beta^{k}$. The conjugates of the Jacobi sum $J_{15}\left(\beta^{m}, \beta^{n}\right)$ are given by $\sigma_{k}\left(J_{15}\left(\beta^{m}, \beta^{n}\right)\right)=J_{15}\left(\beta^{k m}, \beta^{k n}\right)$, where $k=1,2,4,7,8,11,13,14$.

Using the basic properties (2.1), (2.3), (2.4), the nontrivial Jacobi sums $J_{15}\left(\beta^{m}, \beta^{n}\right)$ (where 15 does not divide any of $m, n$ or $m+n$ ) are easily seen to be conjugate to

$$
J\left(\beta, \beta^{n}\right) \quad(n=1,2,3,4,5), \quad J\left(\beta^{3}, \beta^{3}\right), \quad J\left(\beta^{5}, \beta^{5}\right) .
$$

THEOREM 2. Let $p \equiv 1(\bmod 15)$ be a prime. Let $g$ be a primitive root $\bmod p$ and let $\beta=\exp (2 \pi i / 15)$. Then the values of the seven Jacobi sums listed in (4.1) are given by

$$
J_{15}(\beta, \beta)=\sum_{j=0}^{7} b_{j} \beta^{j}
$$

$$
J_{15}\left(\beta, \beta^{2}\right)=\theta \beta^{3 \text { ind } 3}(c+d \sqrt{-15}) \text {, }
$$

$$
J_{15}\left(\beta, \beta^{3}\right)=\beta^{-6 \text { ind } 2-3 \text { ind } 3}(x+u \sqrt{-5-2 \sqrt{5}}+v \sqrt{-5+2 \sqrt{5}}+w \sqrt{5}),
$$

$$
J_{15}\left(\beta, \beta^{4}\right)=\beta^{5 \text { ind } 5}(c+d \sqrt{-15}) \text {, }
$$

$$
J_{15}\left(\beta, \beta^{5}\right)=\theta \beta^{6 \text { ind } 3-5 \text { ind } 5} \sum_{j=0}^{7} b_{j} \beta^{j} \text {, }
$$

(4.8) $J_{15}\left(\beta^{5}, \beta^{5}\right)=\beta^{\text {ind } 2}(a+b \sqrt{-3})$,

where $a, b, c, d, x, u, v, w$ are integers satisfying (1.8), (1.9) and (1.10), and $\theta= \pm 1$ is defined in Theorem 1.

Proof. We begin by examining $\beta^{10 \text { ind } 2} J_{15}\left(\beta^{5}, \beta^{5}\right)$. Clearly, this quantity is of the form $B_{0}+B_{1} \beta^{5}$, where $B_{0}$ and $B_{1}$ are integers. We show that $B_{1}$ is always even. We set $\omega=\beta^{5}=\frac{1}{2}(-1+\sqrt{-3})$ and consider three cases according to the value of ind 2 modulo 3 . 
If ind $2 \equiv 0(\bmod 3)$, then it is known that $(0,0)_{3} \equiv 1(\bmod 2)[1$, Lemma 2$]$. Now as

$$
9(0,0)_{3}=\sum_{m, n=0}^{2} J_{3}\left(\omega^{m}, \omega^{n}\right)
$$

and

$$
J_{3}\left(\omega^{m}, \omega^{n}\right)=J_{15}\left(\beta^{5 m}, \beta^{5 n}\right)= \begin{cases}p-2 & \text { if } m=n=0 \\ B_{0}+B_{1} \omega & \text { if } m=n=1 \\ B_{0}+B_{1} \omega^{2} & \text { if } m=n=2 \\ -1 & \text { otherwise }\end{cases}
$$

we have

$$
9(0,0)_{3}=p-8+2 B_{0}+B_{1}\left(\omega+\omega^{2}\right)=p-8+2 B_{0}-B_{1}
$$

giving

$$
1 \equiv 1-B_{1}(\bmod 2), \quad B_{1} \equiv 0(\bmod 2) .
$$

If ind $2 \equiv 1(\bmod 3)$, then in this case we have $(0,1)_{3} \equiv 1(\bmod 2)[1$, Lemma 2$]$. As

$$
9(0,1)_{3}=\sum_{m, n=0}^{2} J_{3}\left(\omega^{m}, \omega^{n}\right) \omega^{-n}
$$

and

$$
J_{3}\left(\omega^{m}, \omega^{n}\right)=J_{15}\left(\beta^{5 m}, \beta^{5 n}\right)= \begin{cases}p-2 & \text { if } m=n=0 \\ \omega\left(B_{0}+B_{1} \omega\right) & \text { if } m=n=1 \\ \omega^{2}\left(B_{0}+B_{1} \omega^{2}\right) & \text { if } m=n=2 \\ -1 & \text { otherwise, }\end{cases}
$$

we have

$$
9(0,1)_{3}=p-2+2 B_{0}-B_{1},
$$

giving $1 \equiv 1-B_{1}(\bmod 2), B_{1} \equiv 0(\bmod 2)$.

If ind $2 \equiv 2(\bmod 3)$, then in this case we have $(0,2)_{3} \equiv 1(\bmod 2)[1$, Lemma 2$]$. As

$$
9(0,2)_{3}=\sum_{m, n=0}^{2} J_{3}\left(\omega^{m}, \omega^{n}\right) \omega^{n}
$$

and

$$
J_{3}\left(\omega^{m}, \omega^{n}\right)=J_{15}\left(\beta^{5 m}, \beta^{5 n}\right)= \begin{cases}p-2 & \text { if } m=n=0 \\ \omega^{2}\left(B_{0}+B_{1} \omega\right) & \text { if } m=n=1 \\ \omega\left(B_{0}+B_{1} \omega^{2}\right) & \text { if } m=n=2 \\ -1 & \text { otherwise }\end{cases}
$$

we have

$$
9(0,2)_{3}=p-2+2 B_{0}-B_{1}
$$

giving

$$
1 \equiv 1-B_{1}(\bmod 2), \quad B_{1} \equiv 0(\bmod 2)
$$


As $B_{1} \equiv 0(\bmod 2)$ in all three cases, we may define an integer $b$ by $B_{1}=2 b$. Then we have

$$
\beta^{10 \text { ind } 2} J_{15}\left(\beta^{5}, \beta^{5}\right)=B_{0}+2 b\left(\frac{-1+\sqrt{-3}}{2}\right)=a+b \sqrt{-3},
$$

where $a=B_{0}-b$. This completes the proof of (4.8). From (2.2) we have

$$
p=J_{15}\left(\beta^{5}, \beta^{5}\right) J_{15}\left(\beta^{10}, \beta^{10}\right)=\beta^{5 \text { ind } 2}(a+b \sqrt{-3}) \beta^{10 \text { ind } 2}(a-b \sqrt{-3})
$$

so that $p=a^{2}+3 b^{2}$. Cubing (4.8), and using (2.4), we obtain

$$
a \equiv a^{3} \equiv(a+b \sqrt{-3})^{3} \equiv J_{15}\left(\beta^{5}, \beta^{5}\right)^{3} \equiv J_{15}(1,1) \equiv-1(\bmod 3) .
$$

This completes the proof of (1.8).

We next determine $J_{15}\left(\beta^{3}, \beta^{3}\right)$. Appealing to [9, p. 345] we have

$$
J_{15}\left(\beta^{3}, \beta^{3}\right)=\beta^{-6 \text { ind } 2} \sum_{j=0}^{4} t_{j} \beta^{3 j},
$$

where $t_{0}, t_{1}, t_{2}, t_{3}, t_{4}$ are integers such that

$$
\begin{aligned}
& t_{0}+t_{1}+t_{2}+t_{3}+t_{4}=-1, \\
& 5 t_{0}+1=4 x, \\
& t_{1}+t_{2}-t_{3}-t_{4}=4 u, \\
& t_{1}-t_{2}+t_{3}-t_{4}=4 v, \\
& t_{1}-t_{2}-t_{3}+t_{4}=4 \mathrm{w},
\end{aligned}
$$

where $x, u, v, w$ are integers satisfying (1.10). Then, as

$$
\begin{array}{ll}
\beta^{3}=\frac{1}{4}(\sqrt{5}-1+\sqrt{-10-2 \sqrt{5}}), & \beta^{6}=\frac{1}{4}(-\sqrt{5}-1+\sqrt{-10+2 \sqrt{5}}), \\
\beta^{9}=\frac{1}{4}(-\sqrt{5}-1-\sqrt{-10+2 \sqrt{5}}), & \beta^{12}=\frac{1}{4}(\sqrt{5}-1-\sqrt{-10-2 \sqrt{5}}),
\end{array}
$$

we obtain from (4.9)

$$
\begin{aligned}
\beta^{6 \text { ind } 2} J_{15}\left(\beta^{3}, \beta^{3}\right)= & t_{0}-\frac{1}{4}\left(t_{1}+t_{2}+t_{3}+t_{4}\right)+\frac{\sqrt{5}}{4}\left(t_{1}-t_{2}-t_{3}+t_{4}\right) \\
& +\frac{\sqrt{-10-2 \sqrt{5}}}{4}\left(t_{1}-t_{4}\right)+\frac{\sqrt{-10+2 \sqrt{5}}}{4}\left(t_{2}-t_{3}\right) .
\end{aligned}
$$

Now as

$$
\sqrt{-10-2 \sqrt{5}}=\sqrt{-5-2 \sqrt{5}}+\sqrt{-5+2 \sqrt{5}}
$$

and

$$
\sqrt{-10+2 \sqrt{5}}=\sqrt{-5-2 \sqrt{5}}-\sqrt{-5+2 \sqrt{5}}
$$

we obtain, using (4.10)-(4.14),

$$
\begin{aligned}
\beta^{6 \text { ind } 2} J_{15} & \left(\beta^{3}, \beta^{3}\right) \\
= & \frac{1}{4}\left(5 t_{0}+1\right)+\frac{\sqrt{5}}{4}\left(t_{1}-t_{2}-t_{3}+t_{4}\right) \\
& +\frac{1}{4} \sqrt{-5-2 \sqrt{5}}\left(t_{1}+t_{2}-t_{3}-t_{4}\right)+\frac{1}{4} \sqrt{-5+2 \sqrt{5}}\left(t_{1}-t_{2}+t_{3}-t_{4}\right) \\
= & x+u \sqrt{-5-2 \sqrt{5}}+v \sqrt{-5+2 \sqrt{5}}+w \sqrt{5},
\end{aligned}
$$

which is (1.6). 
Next we evaluate $J_{15}\left(\beta, \beta^{3}\right)$. We have

$$
\begin{aligned}
& J_{15}\left(\beta, \beta^{3}\right)=\frac{G_{15}(\beta) G_{15}\left(\beta^{3}\right)}{G_{15}\left(\beta^{4}\right)} \\
& \left.=\theta \beta^{3 \text { ind } 3-5 \text { ind } 5} A C^{2} / B D \quad \text { (by Theorem } 1\right) \\
& =\beta^{-3 \text { ind } 3} \frac{G_{15}\left(\beta^{3}\right)^{2}}{G_{15}\left(\beta^{6}\right)} \quad \text { (by Theorem 1) } \\
& =\beta^{-3 \text { ind } 3} J_{15}\left(\beta^{3}, \beta^{3}\right) \quad(\text { by }(2.8)) \\
& =\beta^{-6 \text { ind } 2-3 \text { ind } 3}(x+u \sqrt{-5-2 \sqrt{5}}+v \sqrt{-5+2 \sqrt{5}}+w \sqrt{5})
\end{aligned}
$$

(by Theorem 1).

This completes the proof of (4.4). The relationship between $J_{15}\left(\beta, \beta^{3}\right)$ and $J_{15}\left(\beta^{3}, \beta^{3}\right)$ was obtained by Dickson [5, p. 198].

Next we show that $\beta^{10 \text { ind } 5} J_{15}\left(\beta, \beta^{4}\right)$ is an element of $Q(\sqrt{-15})$. To do this we must show that it is invariant under $\sigma_{2}$. We have

$$
\begin{aligned}
& \sigma_{2}\left(\beta^{10 \text { ind } 5} J_{15}\left(\beta, \beta^{4}\right)\right)=\beta^{5 \text { ind } 5} J_{15}\left(\beta^{2}, \beta^{8}\right) \\
& =\beta^{\operatorname{sind} 5} \frac{G_{15}\left(\beta^{2}\right) G_{15}\left(\beta^{8}\right)}{G_{15}\left(\beta^{10}\right)} \quad(\text { by }(2.8)) \\
& =\theta \beta^{-3 \text { ind } 3} A B / C \\
& =\beta^{10 \text { ind } 5} \frac{G_{15}(\beta) G_{15}\left(\beta^{4}\right)}{G_{15}\left(\beta^{5}\right)} \quad \text { (by Theorem 1) } \\
& =\beta^{10 \text { ind } 5} J_{15}\left(\beta, \beta^{4}\right) \text {. }
\end{aligned}
$$

As observed by Muskat [14, p. 497], this invariance property follows from the work of Dickson [5, Section 14]. Hence $\beta^{10 \text { ind } 5} J_{15}\left(\beta, \beta^{4}\right)$ is an integer of $Q(\sqrt{-15})$ and so there are rational integers $r$ and $s$ of the same parity such that $\beta^{10 \text { ind } 5} J_{15}\left(\beta, \beta^{4}\right)$ $=\frac{1}{2}(r+s \sqrt{-15})$. From (2.2) we obtain $p=\frac{1}{4}\left(r^{2}+15 s^{2}\right)$. Clearly $r$ and $s$ cannot both be odd, so there are integers $c$ and $d$ such that $r=2 c, s=2 d, p=c^{2}+15 d^{2}$, and $\beta^{10 \text { ind } 5} J_{15}\left(\beta, \beta^{4}\right)=c+d \sqrt{-15}$. This completes the proof of (4.5). The equation (4.5) was give by Muskat in [14, p. 498]. Cubing (4.5), we obtain

$$
c \equiv c^{3} \equiv(c+d \sqrt{-15})^{3} \equiv J_{15}\left(\beta, \beta^{4}\right)^{3} \equiv J_{15}\left(\beta^{3}, \beta^{12}\right) \equiv-1(\bmod 3) .
$$

This completes the proof of (1.9).

Next we show that

$$
J_{15}\left(\beta, \beta^{2}\right)=\theta \beta^{3 \text { ind } 3-5 \text { ind } 5} J_{15}\left(\beta, \beta^{4}\right) .
$$

By (2.8) we have

$$
\frac{J_{15}\left(\beta, \beta^{2}\right)}{J_{15}\left(\beta, \beta^{4}\right)}=\frac{G_{15}\left(\beta^{2}\right) G_{15}\left(\beta^{5}\right)}{G_{15}\left(\beta^{3}\right) G_{15}\left(\beta^{4}\right)} .
$$

Appealing to Theorem 1 , the right-hand side of (4.16) reduces to $\theta \beta^{3 \text { ind } 3-5 \text { ind } 5}$, which proves (4.15). The equation (4.3) then follows from (4.5) and (4.15).

Finally, we show that

$$
J_{15}\left(\beta, \beta^{5}\right)=\theta \beta^{6 \text { ind } 3-5 \text { ind } 5} J_{15}(\beta, \beta) .
$$


By (2.8) and Theorem 1 we have

$$
\frac{J_{15}\left(\beta, \beta^{5}\right)}{J_{15}(\beta, \beta)}=\frac{G_{15}\left(\beta^{2}\right) G_{15}\left(\beta^{5}\right)}{G_{15}(\beta) G_{15}\left(\beta^{6}\right)}=\theta \beta^{6 \text { ind } 3-5 \text { ind } 5} \text {. }
$$

This relationship is given in Dickson [5, p. 199].

This completes the proof of Theorem 2 , as $J_{15}(\beta, \beta)$ can obviously be expressed in the form (4.2), $1, \beta, \ldots, \beta^{7}$ being an integral basis for $Q(\beta)$.

In the next theorem we give Muskat's determination of $\theta$.

Theorem 3 (Muskat [14, Eq. (102)]). For $\theta(= \pm 1)$ as defined in Theorem 1 , we have

$$
\theta \equiv-c(\bmod 5)
$$

Proof. Raising (4.3) to the fifth power, we obtain

$$
-1 \equiv J_{15}\left(\beta^{5}, \beta^{10}\right) \equiv\left(J_{15}\left(\beta, \beta^{2}\right)\right)^{5} \equiv \theta^{5}(c+d \sqrt{-15})^{5} \equiv \theta c(\bmod 5)
$$

as required.

Theorem 3 shows that the value of $\theta$ is given by

$$
\theta=\left\{\begin{array}{cl}
+1 & \text { if } c \equiv-1(\bmod 5) \\
-1 & \text { if } c \equiv 1(\bmod 5)
\end{array}\right.
$$

The same technique enables us to determine ind $10(\bmod 3)$ as well as an alternative determination of $\boldsymbol{\theta}$.

THEOREM 4. With the notation of Theorem 2, we have

$$
\text { ind } 10 \equiv \begin{cases}0(\bmod 3) & \text { if } b \equiv 0(\bmod 5) \\ 1(\bmod 3) & \text { if } a \equiv b(\bmod 5) \\ 2(\bmod 3) & \text { if } a \equiv-b(\bmod 5)\end{cases}
$$

and

$$
\boldsymbol{\theta} \equiv \begin{cases}-a(\bmod 5) & \text { if } b \equiv 0(\bmod 5) \\ 2 a(\bmod 5) & \text { if } b \not \equiv 0(\bmod 5)\end{cases}
$$

Proof. Raising (4.17) to the fifth power, we obtain

$$
\begin{aligned}
-1 & \equiv J_{15}\left(\beta^{5}, \beta^{10}\right) \equiv J_{15}\left(\beta^{5}, \beta^{25}\right) \equiv\left\{J_{15}\left(\beta, \beta^{5}\right)\right\}^{5} \\
& \equiv \theta^{5} \beta^{5 \text { ind } 5}\left\{J_{15}(\beta, \beta)\right\}^{5} \equiv \theta \beta^{5 \text { ind } 5} J_{15}\left(\beta^{5}, \beta^{5}\right) \\
& \equiv \theta \beta^{5 \text { ind } 2+5 \operatorname{ind} 5}(a+b \sqrt{-3})(\bmod 5),
\end{aligned}
$$

that is,

$$
a+b \sqrt{-3} \equiv-\theta \omega^{- \text {ind } 10}(\bmod 5) .
$$

If ind $10 \equiv 0(\bmod 3),(4.21)$ gives

$$
a \equiv-\theta(\bmod 5), \quad b \equiv 0(\bmod 5) .
$$


If ind $10 \equiv 1(\bmod 3),(4.21)$ gives

$$
a \equiv b \equiv \theta / 2(\bmod 5) \text {. }
$$

If ind $10 \equiv 2(\bmod 3),(4.21)$ gives

$$
a \equiv-b \equiv \theta / 2(\bmod 5) .
$$

This completes the proof of Theorem 4.

We note that Theorem 4 gives the following evaluation of $\theta$,

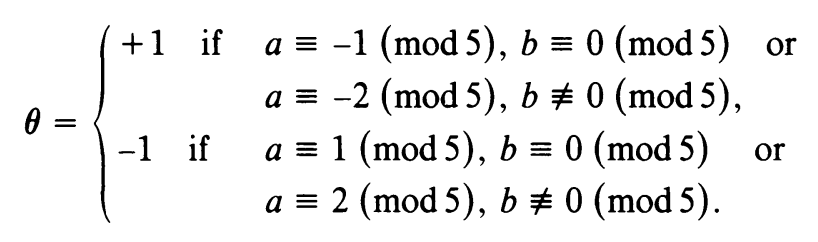

Putting Theorems 3 and 4 together, we get

COROllary 1. With the notation of Theorem 2,

$$
\begin{cases}a \equiv c(\bmod 5) & \text { if } b \equiv 0(\bmod 5) \\ a \equiv 2 c(\bmod 5) & \text { if } b \neq 0(\bmod 5) .\end{cases}
$$

Finally we apply the method once more to obtain ind $18(\bmod 5)$.

THEOREM 5. With the notation of Theorem 2, we have

$$
\text { ind } 18 \equiv \begin{cases}0(\bmod 5) & \text { if } u \equiv v \equiv w \equiv 0(\bmod 3) \\ 1(\bmod 5) & \text { if } x \equiv-u \equiv v \equiv w(\bmod 3) \\ 2(\bmod 5) & \text { if } x \equiv u \equiv v \equiv-w(\bmod 3) \\ 3(\bmod 5) & \text { if }-x \equiv u \equiv v \equiv w(\bmod 3) \\ 4(\bmod 5) & \text { if } x \equiv u \equiv-v \equiv w(\bmod 3)\end{cases}
$$

Proof. Working modulo 3 we have

$$
\begin{aligned}
& \beta^{-6 \text { ind } 2}(x+u \sqrt{-5-2 \sqrt{5}}+v \sqrt{-5+2 \sqrt{5}}+w \sqrt{5}) \\
& \equiv J_{15}\left(\beta^{3}, \beta^{3}\right) \\
& \equiv J_{15}\left(\beta^{3}, \beta^{9}\right) \\
& \equiv\left\{J_{15}\left(\beta, \beta^{3}\right)\right\}^{3} \\
& \equiv \beta^{-3 \text { ind } 2-9 \text { ind } 3}((x+u \sqrt{-5-2 \sqrt{5}}+v \sqrt{-5+2 \sqrt{5}})+w \sqrt{5})^{3} \\
& \text { (by Theorem 2) } \\
& \equiv \beta^{-3 \text { ind } 2-9 \text { ind } 3}(x+u(-5-2 \sqrt{5}) \sqrt{-5-2 \sqrt{5}}+v(-5+2 \sqrt{5}) \\
& \times \sqrt{-5+2 \sqrt{5}}+5 w \sqrt{5})
\end{aligned}
$$

which, using

$$
\sqrt{5} \sqrt{-5-2 \sqrt{5}}=2 \sqrt{-5-2 \sqrt{5}}+\sqrt{-5+2 \sqrt{5}}
$$

and

$$
\sqrt{5} \sqrt{-5+2 \sqrt{5}}=\sqrt{-5-2 \sqrt{5}}-2 \sqrt{-5+2 \sqrt{5}}
$$


gives

$$
\begin{aligned}
x+u \sqrt{-5-2 \sqrt{5}} & +v \sqrt{-5+2 \sqrt{5}}+w \sqrt{5} \\
& \equiv \beta^{3 \text { ind } 18}(x-v \sqrt{-5-2 \sqrt{5}}+u \sqrt{-5+2 \sqrt{5}}-w \sqrt{5})(\bmod 3) .
\end{aligned}
$$

Equating coefficients $(\bmod 3)$ for each residue class of ind $18(\bmod 5)$, we obtain the theorem. We just give the details in the case ind $18 \equiv 2(\bmod 5)$. Replacing $\beta^{6}$ by its expression in terms of radicals, we obtain modulo 3,

$$
\begin{aligned}
& x+u \sqrt{-5-2 \sqrt{5}}+v \sqrt{-5+2 \sqrt{5}}+w \sqrt{5} \\
& \equiv(-\sqrt{5}-1+\sqrt{-5-2 \sqrt{5}}-\sqrt{-5+2 \sqrt{5}}) \\
& \times(x-v \sqrt{-5-2 \sqrt{5}}+u \sqrt{-5+2 \sqrt{5}}-w \sqrt{5}) \\
& \equiv(-x-u-v-w)+(x-u-w) \sqrt{-5-2 \sqrt{5}} \\
& +(-x+u+v) \sqrt{-5+2 \sqrt{5}}+(-x+v+w) \sqrt{5},
\end{aligned}
$$

that is,

$$
x \equiv u \equiv v \equiv-w(\bmod 3) \text {. }
$$

5. Determination of Dickson-Hurwitz $\operatorname{Sums} B_{15}(i, v)$. For any integers $i$ and $v$, the Dickson-Hurwitz sum $B_{15}(i, v)$ is defined by

$$
B_{15}(i, v)=\sum_{h=0}^{14}(h, i-v h)_{15},
$$

where the cyclotomic number $(h, k)_{15}$ was defined in (1.1). The Jacobi sums $J_{15}\left(\beta^{n}, \beta^{n v}\right)$ and the Dickson-Hurwitz sums $B_{15}(i, v)$ are related by the formula (see, for example, [14, p. 489])

$$
J_{15}\left(\beta^{n}, \beta^{n v}\right)=\sum_{i=0}^{14} B_{15}(i, v) \beta^{n i} .
$$

The Dickson-Hurwitz sums have the well-known properties (see, for example, [14, p. 490])

$$
B_{15}(i, v)=B_{15}(i, 14-v)
$$

and

$$
B_{15}(i, 0)=B_{15}(i, 14)= \begin{cases}f-1 & \text { if } 15 \mid i \\ f & \text { if } 15+i\end{cases}
$$

Taking $n=0$ in (5.2), we obtain

$$
\sum_{i=0}^{14} B_{15}(i, v)=p-2 .
$$

From (5.3) and (5.4) we see that the values of all the $15^{2}=225$ Dickson-Hurwitz sums $B_{15}(i, v)(i, v=0,1, \ldots, 14)$ are known, once the values of the $14 \times 7=98$ sums $B_{15}(i, v)(i=0,1, \ldots, 14 ; v=1,2, \ldots, 7)$ have been determined.

In addition, Whiteman [18, Lemma 1] has shown that if $(v, 15)=1$,

$$
B_{15}(i, v)=B_{15}\left(i v^{-1}, v^{-1}\right) \text {, }
$$


where $v v^{-1} \equiv 1(\bmod 15)$. Taking $v=13,2,11$ and 4 in (5.6), and appealing to (5.3) as necessary, we obtain successively

$$
\left\{\begin{array}{l}
B_{15}(i, 1)=B_{15}(7 i, 7) \\
B_{15}(i, 2)=B_{15}(8 i, 6) \\
B_{15}(i, 3)=B_{15}(11 i, 3) \\
B_{15}(i, 4)=B_{15}(4 i, 4)
\end{array}\right.
$$

We now indicate how to form the system of linear equations, which has the 15 Dickson-Hurwitz sums $B_{15}(i, v),(i=0,1,2, \ldots, 14)$ as solutions. The first equation is given by (5.5). The second and third equations are derived by equating the coefficients of 1 and $\sqrt{-3}$ in (5.2) (with $n=5$ ) and appealing to (2.3) and Theorem 2 for the values of $J_{15}\left(\beta^{5}, \beta^{5 v}\right)(v=1, \ldots, 7)$. The fourth, fifth, sixth and seventh equations are obtained by equating the coefficients of $1, \beta^{2}+\beta^{7}, \beta^{3}$ and $\beta^{6}$ in (5.2) (with $n=3)$ and using (2.3) and Theorem 2 for the values of $J_{15}\left(\beta^{3}, \beta^{3 v}\right)(v=$ $1, \ldots, 7)$. The eighth to fifteenth equations are obtained by equating the coefficients of $1, \beta, \beta^{2}, \ldots, \beta^{7}$ in (5.2) (with $n=1$ ) and using (2.3) and Theorem 2 for the values of $J_{15}\left(\beta, \beta^{v}\right)(v=1, \ldots, 7)$. The resulting 15 linear equations in the 15 unknowns $B_{15}(0, v), \ldots, B_{15}(14, v)$ can be expressed in the form

$$
M B(v)=C(v),
$$

where the $15 \times 15$ coefficient matrix $M$ is independent of $v$ and given by

$$
M=\left[\begin{array}{rrrrrrrrrrrrrrr}
1 & 1 & 1 & 1 & 1 & 1 & 1 & 1 & 1 & 1 & 1 & 1 & 1 & 1 & 1 \\
2 & -1 & -1 & 2 & -1 & -1 & 2 & -1 & -1 & 2 & -1 & -1 & 2 & -1 & -1 \\
0 & 1 & -1 & 0 & 1 & -1 & 0 & 1 & -1 & 0 & 1 & -1 & 0 & 1 & -1 \\
1 & 0 & 0 & -1 & 0 & 1 & 0 & 0 & -1 & 0 & 1 & 0 & 0 & -1 & 0 \\
0 & 0 & 0 & 1 & -1 & 0 & 0 & 0 & 1 & -1 & 0 & 0 & 0 & 1 & -1 \\
0 & 1 & 0 & -1 & 0 & 0 & 1 & 0 & -1 & 0 & 0 & 1 & 0 & -1 & 0 \\
0 & 0 & 1 & -1 & 0 & 0 & 0 & 1 & -1 & 0 & 0 & 0 & 1 & -1 & 0 \\
1 & 0 & 0 & 0 & 0 & 0 & 0 & 0 & -1 & -1 & -1 & 0 & 0 & 1 & 1 \\
0 & 1 & 0 & 0 & 0 & 0 & 0 & 0 & 1 & 0 & 0 & -1 & 0 & -1 & 0 \\
0 & 0 & 1 & 0 & 0 & 0 & 0 & 0 & 0 & 1 & 0 & 0 & -1 & 0 & -1 \\
0 & 0 & 0 & 1 & 0 & 0 & 0 & 0 & -1 & -1 & 0 & 0 & 0 & 0 & 1 \\
0 & 0 & 0 & 0 & 1 & 0 & 0 & 0 & 1 & 0 & 0 & 0 & 0 & -1 & -1 \\
0 & 0 & 0 & 0 & 0 & 1 & 0 & 0 & -1 & 0 & -1 & 0 & 0 & 1 & 0 \\
0 & 0 & 0 & 0 & 0 & 0 & 1 & 0 & 0 & -1 & 0 & -1 & 0 & 0 & 1 \\
0 & 0 & 0 & 0 & 0 & 0 & 0 & 1 & 1 & 1 & 0 & 0 & -1 & -1 & -1
\end{array}\right]
$$

and

$$
B(v)=\left[\begin{array}{c}
B_{15}(0, v) \\
B_{15}(1, v) \\
\vdots \\
B_{15}(14, v)
\end{array}\right], \quad C(v)=\left[\begin{array}{c}
C_{1}(v) \\
C_{2}(v) \\
\vdots \\
C_{15}(v)
\end{array}\right],
$$

where the vector $C(v)(v=1, \ldots, 7)$ depends upon $p, a, b, c, d, x, u, v, w$, $b_{0}, \ldots, b_{7}$. The list below gives the number of values taken by each vector $C(v)$ $(v=1, \ldots, 7)$. The numbers of these values depend upon which of the quantities ind $2(\bmod 3)$, ind $2(\bmod 5)$, ind $3(\bmod 5)$, ind $5(\bmod 3)$, and $c(\bmod 5)$ are involved. 


\begin{tabular}{ccl}
\hline$v$ & $\begin{array}{c}\text { number of values } \\
\text { of } C(v)\end{array}$ & \multicolumn{1}{c}{ parameters involved } \\
\hline 1 & 15 & ind $2(\bmod 3)$, ind $2(\bmod 5)$ \\
2 & 50 & ind $2(\bmod 5)$, ind $3(\bmod 5), c(\bmod 5)$ \\
3 & 25 & ind $2(\bmod 5), \operatorname{ind} 3(\bmod 5)$ \\
4 & 9 & ind $2(\bmod 3)$, ind $5(\bmod 3)$ \\
5 & 30 & ind $3(\bmod 5)$, ind $5(\bmod 3), c(\bmod 5)$ \\
6 & 50 & ind $2(\bmod 5)$, ind $3(\bmod 5), c(\bmod 5)$ \\
7 & 15 & \\
\hline
\end{tabular}

Thus there are 194 cases to be considered. However, to save space, we,will not give the values of the vectors $C(v)$ here.

Inverting the matrix $M$ in (5.9), the matrix equation (5.8) becomes

$$
30 B(v)=N C(v), \quad v=1,2, \ldots, 7,
$$

where

$$
N=\left[\begin{array}{rrrrrrrrrrrrrrr}
2 & 2 & 0 & 8 & 2 & -2 & -2 & 16 & 2 & 2 & -4 & 2 & -8 & -4 & 2 \\
2 & -1 & 3 & -2 & 2 & 8 & -2 & 2 & 16 & 2 & 2 & -4 & 2 & -8 & -4 \\
2 & -1 & -3 & -2 & 2 & -2 & 8 & 2 & 2 & 16 & 2 & 2 & -4 & 2 & -8 \\
2 & 2 & 0 & -2 & 2 & -2 & -2 & -4 & 2 & 2 & 16 & 2 & 2 & -4 & 2 \\
2 & -1 & 3 & -2 & -8 & -2 & -2 & 2 & -4 & 2 & 2 & 16 & 2 & 2 & -4 \\
2 & -1 & -3 & 8 & 2 & -2 & -2 & -8 & 2 & -4 & 2 & 2 & 16 & 2 & 2 \\
2 & 2 & 0 & -2 & 2 & 8 & -2 & -4 & -8 & 2 & -4 & 2 & 2 & 16 & 2 \\
2 & -1 & 3 & -2 & 2 & -2 & 8 & 2 & -4 & -8 & 2 & -4 & 2 & 2 & 16 \\
2 & -1 & -3 & -2 & 2 & -2 & -2 & 2 & 2 & -4 & -8 & 2 & -4 & 2 & 2 \\
2 & 2 & 0 & -2 & -8 & -2 & -2 & -4 & 2 & 2 & -4 & -8 & 2 & -4 & 2 \\
2 & -1 & 3 & 8 & 2 & -2 & -2 & -8 & -4 & 2 & 2 & -4 & -8 & 2 & -4 \\
2 & -1 & -3 & -2 & 2 & 8 & -2 & 2 & -8 & -4 & 2 & 2 & -4 & -8 & 2 \\
2 & 2 & 0 & -2 & 2 & -2 & 8 & -4 & 2 & -8 & -4 & 2 & 2 & -4 & -8 \\
2 & -1 & 3 & -2 & 2 & -2 & -2 & 2 & -4 & 2 & -8 & -4 & 2 & 2 & -4 \\
2 & -1 & -3 & -2 & -8 & -2 & -2 & 2 & 2 & -4 & 2 & -8 & -4 & 2 & 2
\end{array}\right] .
$$

The calculation of the $15 \times 1$ vector $N C(v)$ in each of the 194 cases indicated above was then carried out on a Digital Professional 350 microcomputer to obtain the values of the Dickson-Hurwitz sums $B_{15}(i, v)(i=0,1, \ldots, 14 ; v=1,2, \ldots, 7)$. In view of (5.7) it is only necessary to have the values of

$$
\begin{cases}B_{15}(i, v) & (i=0,1, \ldots, 14 ; v=1,2,5), \\ B_{15}(i, 3) & (i=0,1,2,3,4,5,6,8,9,12), \\ B_{15}(i, 4) & (i=0,1,2,3,5,6,7,10,11)\end{cases}
$$

to be able to deduce the values of all of them. In order to save space we do not list the values of these sums here.

6. Calculation of the Cyclotomic Numbers $(h, k)_{15}$. The cyclotomic numbers $(h, k)_{15}$ have the properties (see, for example, $[18$, p. 96])

$$
(h, k)_{15}=(k, h)_{15}=(15-h, k-h)_{15} .
$$


Appealing to (6.1), we see that each of the $15^{2}=225$ cyclotomic numbers $(h, k)_{15}$ is equal to one of the 46 cyclotomic numbers

$$
\begin{cases}(0, k)_{15}, & k=0,1, \ldots, 14, \\ (1, k)_{15}, & k=2, \ldots, 13, \\ (2, k)_{15}, & k=4, \ldots, 12, \\ (3, k)_{15}, & k=6, \ldots, 11, \\ (4, k)_{15}, & k=8,9,10, \\ (5,10)_{15} . & \end{cases}
$$

The relationships between the cyclotomic numbers are exhibited in the matrix near the end of this section, in which $(h, k)_{15}$ is in row $h(0 \leqslant h \leqslant 14)$ and column $k$ $(0 \leqslant k \leqslant 14)$.

In order to determine explicit formulae for the cyclotomic numbers in (6.2), we express each cyclotomic number in terms of the Dickson-Hurwitz sums $B_{15}(i, v)$ and then appeal to the formulae for these sums derived in Section 5. These calculations were performed on a Digital Professional 350 microcomputer.

The formula used to express the cyclotomic numbers in terms of the DicksonHurwitz sums is given in Theorem 6 below. This formula is the special case $e=15$, $x=3, y=5, q=p$ of Theorem 7 in [8], simplified using (5.7).

THEOREM 6. Let $p=15 f+1$ be prime and set $\delta_{r}=1$, if $r \equiv 0(\bmod 15)$, and $\delta_{r}=0$, if $r \not \equiv 0(\bmod 15)$. Then we have

$$
\begin{aligned}
3375(r, s)_{15}= & 226 p-451-225\left(\delta_{r}+\delta_{s}+\delta_{r-s}\right) \\
& +30\left(\delta_{5 r}+\delta_{5 s}\right)+60\left(\delta_{3 r}+\delta_{3 s}\right) \\
& +S_{1}+S_{2}+S_{3}+S_{4}+S_{5},
\end{aligned}
$$

where

$$
\begin{aligned}
S_{1}= & 15 \cdot 15 B_{15}(r+s, 1) \\
& +7\left(15 B_{15}(r+13 s, 1)+15 B_{15}(13 r+s, 1)\right) \\
& -3 \sum_{t=1}^{4}\left(15 B_{15}(r+13 s+3 t, 1)+15 B_{15}(13 r+s+3 t, 1)\right) \\
& -5 \sum_{t=1}^{2}\left(15 B_{15}(r+13 s+5 t, 1)+15 B_{15}(13 r+s+5 t, 1)\right) \\
S_{2}= & 7\left(15 B_{15}(r+2 s, 2)+15 B_{15}(r+12 s, 2)+15 B_{15}(12 r+2 s, 2)\right. \\
+ & \left.15 B_{15}(2 r+s, 2)+15 B_{15}(12 r+s, 2)+15 B_{15}(2 r+12 s, 2)\right) \\
- & 3 \sum_{t=1}^{4}\left(15 B_{15}(r+2 s+3 t, 2)+15 B_{15}(r+12 s+3 t, 2)\right. \\
& +15 B_{15}(12 r+2 s+3 t, 2)+15 B_{15}(2 r+s+3 t, 2) \\
& \left.+15 B_{15}(12 r+s+3 t, 2)+15 B_{15}(2 r+12 s+3 t, 2)\right)
\end{aligned}
$$




$$
\begin{aligned}
& -5 \sum_{t=1}^{2}\left(15 B_{15}(r+2 s+5 t, 2)+15 B_{15}(r+12 s+5 t, 2)\right. \\
& +15 B_{15}(12 r+2 s+5 t, 2)+15 B_{15}(2 r+s+5 t, 2) \\
& \left.+15 B_{15}(12 r+s+5 t, 2)+15 B_{15}(2 r+12 s+5 t, 2)\right) \\
& -5 \sum_{t=0}^{2}\left(15 B_{15}(2 r+s+5 t, 2)+15 B_{15}(r+2 s+5 t, 2)\right) \text {, } \\
& S_{3}=7\left(15 B_{15}(r+3 s, 3)+15 B_{15}(r+11 s, 3)+15 B_{15}(3 r+s, 3)\right) \\
& -3 \sum_{t=1}^{4}\left(15 B_{15}(r+3 s+3 t, 3)+15 B_{15}(r+11 s+3 t, 3)\right. \\
& \left.+15 B_{15}(3 r+s+3 t, 3)\right) \\
& -5 \sum_{t=1}^{2}\left(15 B_{15}(r+3 s+5 t, 3)+15 B_{15}(r+11 s+5 t, 3)\right. \\
& \left.+15 B_{15}(3 r+s+5 t, 3)\right) \\
& S_{4}=7\left(15 B_{15}(r+4 s, 4)+15 B_{15}(r+10 s, 4)+15 B_{15}(10 r+s, 4)\right) \\
& -3 \sum_{t=1}^{4}\left(15 B_{15}(r+4 s+3 t, 4)+15 B_{15}(r+10 s+3 t, 4)\right. \\
& \left.+15 B_{15}(10 r+s+3 t, 4)\right) \\
& -5 \sum_{t=1}^{2}\left(15 B_{15}(r+4 s+5 t, 4)+15 B_{15}(r+10 s+5 t, 4)\right. \\
& \left.+15 B_{15}(10 r+s+5 t, 4)\right), \\
& S_{5}=7\left(15 B_{15}(r+5 s, 5)+15 B_{15}(r+9 s, 5)+15 B_{15}(5 r+s, 5)\right. \\
& \left.+15 B_{15}(9 r+s, 5)\right) \\
& +13\left(15 B_{15}(5 r+9 s, 5)+15 B_{15}(9 r+5 s, 5)\right) \\
& -3 \sum_{t=1}^{4}\left(15 B_{15}(r+5 s+3 t, 5)+15 B_{15}(r+9 s+3 t, 5)\right. \\
& \left.+15 B_{15}(5 r+s+3 t, 5)+15 B_{15}(9 r+s+3 t, 5)\right) \\
& -5 \sum_{t=1}^{2}\left(15 B_{15}(r+5 s+5 t, 5)+15 B_{15}(r+9 s+5 t, 5)\right. \\
& \left.+15 B_{15}(5 r+s+5 t, 5)+15 B_{15}(9 r+s+5 t, 5)\right) \\
& -\sum_{t=1}^{4}\left(15 B_{15}(5 r+9 s+3 t, 5)+15 B_{15}(9 r+5 s+3 t, 5)\right) \\
& -\sum_{t=1}^{2}\left(15 B_{15}(5 r+9 s+5 t, 5)+15 B_{15}(9 r+5 s+5 t, 5)\right) .
\end{aligned}
$$

The resulting expressions for the cyclotomic numbers (6.2) are given in Tables $1-70$. These tables are given on microfiche cards inserted inside the back cover of this issue. Each number $225(h, k)_{15}$ is given as an integral linear combination of $p$, 
$1, a, b, c, d, x, u, v, w, b_{0}, b_{1}, b_{2}, b_{3}, b_{4}, b_{5}, b_{6}, b_{7}$. Not all 450 cases are given, as they can be deduced from the given tables by replacing the primitive root $g$ by the primitive root $g^{m}$, where $(m, p-1)=1$, for a suitable value of $m$.

It is easy to check that the effect of this replacement is as follows:

$$
\begin{aligned}
& \text { ind } k \rightarrow m \text { ind } k, \quad k=2,3,5, \\
& (r, s)_{15} \rightarrow(m r, m s)_{15}, \\
& (a, b) \rightarrow \begin{cases}(a, b) & \text { if } m \equiv 1(\bmod 3), \\
(a,-b) & \text { if } m \equiv 2(\bmod 3),\end{cases} \\
& (c, d) \rightarrow \begin{cases}(c, d) & \text { if } m \equiv 1,2,4,8(\bmod 15), \\
(c,-d) & \text { if } m \equiv 7,11,13,14(\bmod 15),\end{cases} \\
& (x, u, v, w) \rightarrow \begin{cases}(x, u, v, w) & \text { if } m \equiv 1(\bmod 5), \\
(x,-v, u,-w) & \text { if } m \equiv 2(\bmod 5), \\
(x, v,-u,-w) & \text { if } m \equiv 3(\bmod 5), \\
(x,-u,-v, w) & \text { if } m \equiv 4(\bmod 5),\end{cases}
\end{aligned}
$$

and that

$$
\left[\begin{array}{c}
b_{0} \\
b_{1} \\
\vdots \\
b_{7}
\end{array}\right]
$$

is transformed as follows:

$$
\begin{array}{clll}
\frac{m \equiv 1(\bmod 15)}{b_{0}} & \frac{m \equiv 2(\bmod 15)}{b_{0}-b_{1}-b_{3}-b_{5}} & \frac{m \equiv 4(\bmod 15)}{b_{0}-b_{2}-b_{6}+b_{7}} & \frac{m \equiv 7(\bmod 15)}{b_{0}+b_{1}-b_{3}} \\
b_{1} & b_{1}+b_{2}-b_{7} & b_{2}+b_{4}-b_{7} & -b_{1}-b_{2}+b_{7} \\
b_{2} & b_{3}+b_{4} & -b_{3}+b_{6} & b_{3} \\
b_{3} & -b_{1}-b_{3}+b_{6} & -b_{2}-b_{6} & -b_{3}+b_{6} \\
b_{4} & b_{1} & b_{1}+b_{2}-b_{7} & -b_{1} \\
b_{5} & -b_{1}-b_{5} & -b_{2}+b_{5}+b_{7} & b_{1}+b_{5} \\
b_{6} & -b_{3}-b_{7} & -b_{6} & -b_{2}-b_{3} \\
b_{7} & b_{1}+b_{3} & b_{2}-b_{3}+b_{6}-b_{7} & -b_{1}+b_{3}+b_{4} \\
& & & \\
m \equiv 8(\bmod 15) & m \equiv 11(\bmod 15) & \frac{m \equiv 13(\bmod 15)}{b_{0}+b_{2}+b_{4}} & \frac{m \equiv 14(\bmod 15)}{b_{0}+b_{1}+b_{2}-b_{5}-b_{6}-b_{7}} \\
\frac{m}{b_{0}-b_{4}-b_{5}+b_{7}} & b_{0}+b_{4}-b_{5} & -b_{4} & -b_{2}-b_{4}+b_{7} \\
b_{4} & -b_{1} & -b_{2}-b_{6} & -b_{1}-b_{3}+b_{6} \\
b_{1}-b_{6}-b_{7} & -b_{4}+b_{7} & +b_{2} & b_{1}-b_{6}-b_{7} \\
-b_{4}+b_{7} & b_{3}+b_{4} & -b_{2}-b_{4}+b_{7} & -b_{1}-b_{2}+b_{7} \\
b_{2}+b_{4}-b_{7} & -b_{4} & b_{4}+b_{5} & b_{2}-b_{5}-b_{7} \\
-b_{4}-b_{5} & -b_{5} & b_{2}+b_{3} & b_{1}-b_{4}-b_{6} \\
b_{3}+b_{7} & -b_{1}+b_{4}+b_{6} & b_{1}-b_{2}-b_{4}-b_{6} & -b_{1}-b_{2}-b_{3}+b_{6}+b_{7} \\
b_{4}-b_{6}-b_{7} & b_{2}-b_{4} &
\end{array}
$$

Finally, we show how to find $(2,4)_{15}$ when

$$
\begin{aligned}
& \text { ind } 2 \equiv 0(\bmod 3), \quad \text { ind } 2 \equiv 1(\bmod 5), \quad \text { ind } 3 \equiv 0(\bmod 5), \\
& \text { ind } 5 \equiv 2(\bmod 3), \quad c \equiv-1(\bmod 5) .
\end{aligned}
$$


We use the formula for $(1,8)_{15}$ given in Table 12 , as replacing $g$ by $g^{m}$, where $(m, p-1)=1$ and $m \equiv 11(\bmod 15)$, in the parameters specifying Table 12 gives $(6.3)$, and $(1,8)_{15} \rightarrow(11,88)_{15}=(11,13)_{15}=(2,4)_{15}$. Replacing

$$
\left(a, b, c, d, x, u, v, w, b_{0}, b_{1}, b_{2}, b_{3}, b_{4}, b_{5}, b_{6}, b_{7}\right)
$$

by

$$
\begin{aligned}
\left(a,-b, c,-d, x, u, v, w, b_{0}+b_{4}-b_{5},-b_{1},-b_{4}+b_{7}\right. \\
\left.b_{3}+b_{4},-b_{4},-b_{5},-b_{1}+b_{4}+b_{6}, b_{2}-b_{4}\right)
\end{aligned}
$$

in

$$
\begin{aligned}
225(1,8)_{15}= & p+1+2 a-c-15 d-5 x+5 u+15 v \\
& -5 w+b_{0}+5 b_{1}-b_{2}+11 b_{3}-10 b_{4}-4 b_{5}-4 b_{6}+20 b_{7},
\end{aligned}
$$

we obtain

$$
\begin{aligned}
225(2,4)_{15}= & p+1+2 a-c+15 d-5 x+5 u+15 v \\
& -5 w+b_{0}-b_{1}+20 b_{2}+11 b_{3}-b_{4}-5 b_{5}-4 b_{6}-b_{7} .
\end{aligned}
$$

As a check on the correctness of the tables, the values of $a, b, c, d, x, u, v, w, b_{0}$, $b_{1}, \ldots, b_{7}$ were calculated for 150 pairs $(p, g)$, where $p$ is a prime congruent to 1 modulo 15 in the range $31 \leqslant p \leqslant 5581$ and $g$ is a primitive root modulo $p$. For each pair $(p, g)$ a cyclotomic number was chosen at random and its value calculated from the appropriate table. The resulting value was then compared with the value of the cyclotomic number computed directly. In every case the two values agreed.

For example, using the values of $a, b, c, d, x, u, v, w, b_{0}, b_{1}, \ldots, b_{7}$ given in Section 1 for $p=61, g=2$ in Table 50, we find that of the 46 cyclotomic numbers listed in (6.2) only the following are nonzero:

$$
\begin{aligned}
(0,1)_{15} & =(1,6)_{15}=(1,10)_{15}=(1,12)_{15}=(2,4)_{15}=(2,6)_{15} \\
& =(2,7)_{15}=(3,6)_{15}=(3,11)_{15}=(5,10)_{15}=1, \\
(0,8)_{15} & =2 .
\end{aligned}
$$

These values are easily checked by direct calculation.

Relationships among cyclotomic numbers $(h, k)_{15}$

$\begin{array}{llllllllllllllll} & 0 & 1 & 2 & 3 & 4 & 5 & 6 & 7 & 8 & 9 & 10 & 11 & 12 & 13 & 14 \\ 0 & 0,0 & 0,1 & 0,2 & 0,3 & 0,4 & 0,5 & 0,6 & 0,7 & 0,8 & 0,9 & 0,10 & 0,11 & 0,12 & 0,13 & 0,14 \\ 1 & 0,1 & 0,14 & 1,2 & 1,3 & 1,4 & 1,5 & 1,6 & 1,7 & 1,8 & 1,9 & 1,10 & 1,11 & 1,12 & 1,13 & 1,2 \\ 2 & 0,2 & 1,2 & 0,13 & 1,13 & 2,4 & 2,5 & 2,6 & 2,7 & 2,8 & 2,9 & 2,10 & 2,11 & 2,12 & 2,4 & 1,3 \\ 3 & 0,3 & 1,3 & 1,13 & 0,12 & 1,12 & 2,12 & 3,6 & 3,7 & 3,8 & 3,9 & 3,10 & 3,11 & 3,6 & 2,5 & 1,4 \\ 4 & 0,4 & 1,4 & 2,4 & 1,12 & 0,11 & 1,11 & 2,11 & 3,11 & 4,8 & 4,9 & 4,10 & 4,8 & 3,7 & 2,6 & 1,5 \\ 5 & 0,5 & 1,5 & 2,5 & 2,12 & 1,11 & 0,10 & 1,10 & 2,10 & 3,10 & 4,10 & 5,10 & 4,9 & 3,8 & 2,7 & 1,6 \\ 6 & 0,6 & 1,6 & 2,6 & 3,6 & 2,11 & 1,10 & 0,9 & 1,9 & 2,9 & 3,9 & 4,9 & 4,10 & 3,9 & 2,8 & 1,7 \\ 7 & 0,7 & 1,7 & 2,7 & 3,7 & 3,11 & 2,10 & 1,9 & 0,8 & 1,8 & 2,8 & 3,8 & 4,8 & 3,10 & 2,9 & 1,8 \\ 8 & 0,8 & 1,8 & 2,8 & 3,8 & 4,8 & 3,10 & 2,9 & 1,8 & 0,7 & 1,7 & 2,7 & 3,7 & 3,11 & 2,10 & 1,9 \\ 9 & 0,9 & 1,9 & 2,9 & 3,9 & 4,9 & 4,10 & 3,9 & 2,8 & 1,7 & 0,6 & 1,6 & 2,6 & 3,6 & 2,11 & 1,10 \\ 10 & 0,10 & 1,10 & 2,10 & 3,10 & 4,10 & 5,10 & 4,9 & 3,8 & 2,7 & 1,6 & 0,5 & 1,5 & 2,5 & 2,12 & 1,11 \\ 11 & 0,11 & 1,11 & 2,11 & 3,11 & 4,8 & 4,9 & 4,10 & 4,8 & 3,7 & 2,6 & 1,5 & 0,4 & 1,4 & 2,4 & 1,12 \\ 12 & 0,12 & 1,12 & 2,12 & 3,6 & 3,7 & 3,8 & 3,9 & 3,10 & 3,11 & 3,6 & 2,5 & 1,4 & 0,3 & 1,3 & 1,13 \\ 13 & 0,13 & 1,13 & 2,4 & 2,5 & 2,6 & 2,7 & 2,8 & 2,9 & 2,10 & 2,11 & 2,12 & 2,4 & 1,3 & 0,2 & 1,2 \\ 14 & 0,14 & 1,2 & 1,3 & 1,4 & 1,5 & 1,6 & 1,7 & 1,8 & 1,9 & 1,10 & 1,11 & 1,12 & 1,13 & 1,2 & 0,1\end{array}$


Department of Mathematics

College of New Caledonia

Prince George, B. C., Canada V2N 1 P8

Department of Mathematics and Statistics

Carleton University

Ottawa, Ontario, Canada K1S 5B6

Department of Mathematics

Okanagan College

Vernon, B. C., Canada V1B 2N5

Department of Mathematics and Statistics

Carleton University

Ottawa, Ontario, Canada K1S 5B6

1. H. P. Alderson, "On the septimic character of 2 and 3," Proc. Cambridge Philos. Soc., v. 74, 1973, pp. 421-433.

2. L. D. BAUMert \& H. FredrickSEN, “The cyclotomic numbers of order eighteen with applications to difference sets," Math. Comp., v. 21, 1967, pp. 204-219.

3. H. DAVENPORT \& H. HASSE, "Die Nullstellen der Kongruenzzetafunktionen in gewissen zyklischen Fällen,” J. Reine Angew. Math., v. 172, 1934, pp. 151-182.

4. L. E. Dickson, "Cyclotomy, higher congruences, and Waring's problem," Amer. J. Math., v. 57, 1935, pp. 391-424.

5. L. E. Dickson, "Cyclotomy when $e$ is composite," Trans. Amer. Math. Soc., v. 38, 1935, pp. $187-200$.

6. R. J. Evans \& J. R. Hill, “The cyclotomic numbers of order sixteen,” Math. Comp., v. 33, 1979, pp. 827-835.

7. R. J. Evans, “Table of cyclotomic numbers of order twenty-four," Math. Comp.,v. 35, 1980, Review 12, pp. 1036-1038.

8. C. Friesen, J. B. Muskat, B. K. Spearman \& K. S. Williams, "Cyclotomy of order 15 over $\mathrm{GF}\left(p^{2}\right), p \equiv 4,11(\bmod 15), "$ Internat. J. Math. Math. Sci. (To appear.)

9. R. E. Guidici, J. B. Muskat \& S. F. Robinson, "On the evaluation of Brewer's character sums," Trans. Amer. Math. Soc., v. 171, 1972, pp. 317-347.

10. E. Lehmer, "On the number of solutions of $u^{k}+D \equiv w^{2}(\bmod p)$," Pacific J. Math., v. 5, 1955 , pp. $103-118$.

11. P. A. Leonard \& K. S. Williams, "The cyclotomic numbers of order seven," Proc. Amer. Math. Soc., v. 51, 1975, pp. 295-300.

12. P. A. LeONARD \& K. S. Williams, “The cyclotomic numbers of order eleven," Acta Arith., v. 26, 1975 , pp. $367-383$.

13. J. B. MUSKAT, "The cyclotomic numbers of order fourteen," Acta Arith., v. 11, 1966, pp. 263-279.

14. J. B. MuSKat, "On Jacobi sums of certain composite orders," Trans. Amer. Math. Soc., v. 134, 1968 , pp. 483-502.

15. J. B. Muskat \& A. L. Whiteman, “The cyclotomic numbers of order twenty”, Acta Arith., v. 17, 1970, pp. 185-216.

16. J. B. MUSKAT \& Y.- C. ZEE, "On the uniqueness of solutions of certain diophantine equations," Proc. Amer. Math. Soc., v. 49, 1975, pp. 13-19.

17. A. L. Whiteman, "The cyclotomic numbers of order sixteen," Trans. Amer. Math. Soc., v. 86, 1957, pp. 401-413.

18. A. L. Whiteman, The Cyclotomic Numbers of Order Ten, Proc. Sympos. Appl. Math., vol. 10, Amer. Math. Soc., Providence, R. I., 1960, pp. 95-111.

19. A. L. Whiteman, “The cyclotomic numbers of order twelve," Acta Arith., v. 6, 1960, pp. 53-76. 